\title{
Location of colorectal cancer: colonoscopy versus surgery. Yield of colonoscopy in predicting actual location
}

\section{다)(1) $\odot$}

Authors

Juan Pablo Blum-Guzman, Silvio Wanderley de Melo Jr.

Institution

University of Florida College of Medicine Jacksonville, Jacksonville, Florida, United States

submitted 21.6.2016

accepted after revision 17.1.2017

\section{Bibliography}

DOI https://doi.org/10.1055/s-0043-110564 |

Endoscopy International Open 2017; 05: E642-E645

(c) Georg Thieme Verlag KG Stuttgart · New York

ISSN 2364-3722

Corresponding author

Silvio Wanderley de Melo Junior, MD, 4555 Emerson Street, Suite 300, Jacksonville, FL, 32207

Fax: +1-904-633-0028

Silvio.demelo@jax.ufl.edu

\section{ABSTRACT}

Background and study aims Recent studies suggest that differences in biological characteristics and risk factors across cancer site within the colon and rectum may translate to differences in survival. It can be challenging at times to determine the precise anatomical location of a lesion with a luminal view during colonoscopy. The aim of this study is to determine if there is a significant difference between the location of colorectal cancers described by gastroenterologists in colonoscopies and the actual anatomical location noted on operative and pathology reports after colon surgery.

Patients and methods A single-center retrospective analysis of colonoscopies of patient with reported colonic masses from January 2005 to April $2014(n=380)$ was carried. Assessed data included demography, operative and pathology reports. Findings were compared: between the location of colorectal cancers described by gastroenterologists in colonoscopies and the actual anatomical location noted on operative reports or pathology samples.

Results We identified 380 colonic masses, 158 were confirmed adenocarcinomas. Of these 123 underwent surgical resection, 27 had to be excluded since no specific location was reported on their operative or pathology report. An absolute difference between endoscopic and surgical location was found in 32 cases (33\%). Of these, 22 (23\%) differed by 1 colonic segment, $8(8 \%)$ differed by 2 colonic segments and 2 (2\%) differed by 3 colonic segments.

Conclusion There is a significant difference between the location of colorectal cancers reported by gastroenterologists during endoscopy and the actual anatomical location noted on operative or pathology reports after colon surgery. Endoscopic tattooing should be used when faced with any luminal lesions of interest.

\section{Introduction}

Colorectal cancer has been suggested to be a multi-pathway disease based on different biomolecular properties [1-3]. Some of these characteristics are more frequent in certain locations, such as high microsatellite instability in tumors of the right colon. Thus, there could also be differences in carcinogenesis and pathology between different parts of the bowel. For example, family history is more strongly associated with risk of proximal colon cancer than rectal cancer, and alcohol consumption is more strongly associated with risk of rectal cancer than colon cancer. Cancers in the proximal colon are more likely than cancers in the distal colon and rectum to be diagnosed in women and to be diagnosed at a later age [4]. Recent studies suggest that differences in biological characteristics and risk factors across cancer site within the colon and rectum may translate to differences in survival. In particular, proximal colon cancer has been associated with poorer survival than distal coIon cancer, but there appears to be little difference in survival for cancers arising in the distal colon versus rectum [5].

Colonoscopy is widely recommended for early detection and prevention of colorectal cancer based on observational and modeling studies [6]. However, it can be challenging at times to determine the precise anatomical location of a lesion with a luminal view during colonoscopy. We hypothesize in our study that there is a significant difference between the location of colorectal cancers described by gastroenterologists in colonoscopies and the actual anatomical location noted on operative and pathology reports after colon resection surgery. 
- Table 1 Demographic characteristics.

\begin{tabular}{|l|c|c|}
\hline Variable & Frequency & Percent \\
\hline Race & & \\
\hline - Caucasians & 58 & 47.15 \\
\hline - African Americans & 59 & 47.97 \\
\hline - Other & 5 & 4.88 \\
\hline Gender & & \\
\hline - Female & 68 & 55.28 \\
\hline - Male & 55 & 44.72 \\
\hline Age (mean \pm SD) & $65.0 \pm 12.7$ & \\
\hline
\end{tabular}

\section{Patients and methods}

We reviewed colonoscopy reports from January 2005 to April 2014 and identified those which described colonic masses. We reviewed pathology reports of biopsies taken from described masses and confirmed those reported as adenocarcinomas. We collected patient demographics including age, gender and ethnicity. We then revised operative reports and pathology reports from those patients that underwent surgical resection.

We compared findings between the location of colorectal cancers described by gastroenterologists during colonoscopies, the anatomical location noted on operative and pathology reports after colon surgery. We classified them according to the colonic segment reported: cecum, ascending colon, hepatic flexure, transverse colon, splenic flexure, descending colon, sigmoid colon, or rectum. Categorical variables were summarized using counts and percentages, and continuous variables were summarized using means and standard deviations. Three comparisons listed below were performed: 1 . between the location of colorectal cancers described by gastroenterologists in colonoscopies and the actual anatomical location noted on operative reports by surgeons (endo-surgery); 2 . between the location of colorectal cancers described by gastroenterologists in colonoscopies and the location noted by pathology (endopathology); and 3. Between the actual anatomical location noted on operative reports by surgeons and the location noted by pathology (surgery-pathology). The weighted Kappa coefficient was calculated, along with its $95 \%$ confidence interval, to estimate the agreement between the two strategies. Then, the absolute difference between the locations described by the two procedures was calculated for each tumor.

\section{Results}

We identified 380 colonic masses, of which 158 were confirmed adenocarcinomas. Of these 123 underwent surgical resection. We had a slight female predominance of 63 patients (55.3\%) versus 55 males (44.7\%). We had equal distribution between Caucasians, 58 (47\%), and African Americans, 59 (48\%) with 5 $(5 \%)$ as other races. The mean age was $65 \pm 12.7$. A summary of the patient characteristics is shown in $>$ Table 1 . Of these 123
Table 2 Location of colonic adenocarcinomas.

\begin{tabular}{|l|r|r|r|}
\hline Colonic segment & Endoscopy & Surgery & Pathology \\
\hline Cecum & 18 & 13 & 30 \\
\hline Ascending & 30 & 11 & 20 \\
\hline Hepatic flexure & 9 & 8 & 3 \\
\hline Transverse & 16 & 16 & 15 \\
\hline Splenic flexure & 3 & 5 & 2 \\
\hline Descending & 3 & 2 & 2 \\
\hline Sigmoid & 21 & 18 & 19 \\
\hline Rectum & 24 & 24 & 22 \\
\hline Total & 124 & 97 & 113 \\
\hline
\end{tabular}

cases, 27 had to be excluded since no specific location was reported on their operative or pathology report.

The location of colonic adenocarcinomas is summarized in - Table 2. A total of 124 confirmed colon adenocarcinomas were found on endoscopy reports of 123 patients who underwent surgical resection. Two lesions were found in 2 different colonic segments in 1 patient. Ninety-six operative reports described the anatomical location of the colonic masses, $27 \mathrm{did}$ not report a specific location. Of the 123 pathology reports reviewed, 112 of them were able to specify the colonic segment were the lesion was found.

The difference between endoscopic and surgical location was $33 \%$ (32 cases). When dividing the colon into 8 segments (cecum, ascending colon, hepatic flexure, transverse colon, splenic flexure, descending colon, sigmoid colon, and rectum), 64 cases $(67 \%)$ had the same location by colonoscopy and the operative note, 22 cases (23\%) differed by one colonic segment, 8 cases $(8 \%)$ differed by two colonic segments and 2 cases (2\%) differed by 3 colonic segments.

Overall, colonoscopic and surgical localization had an almost perfect agreement with a weighted Kappa coefficient of 0.843 (95\% Cl, 0.079-0.896) [7].

The difference in localization between endoscopic and pathology was $30 \%$ (34 cases). When dividing the colon into 8 segments (cecum, ascending colon, hepatic flexure, transverse colon, splenic flexure, descending colon, sigmoid colon, and rectum), 78 cases (70\%) had the same location by colonoscopy and the pathology report, 26 (23\%) differed by 1 colonic segment, 5 (4\%) differed by 2 colonic segments, 2 (2\%) differed by 3 colonic segments and 1 (1\%) differed by 5 colonic segments.

Once again, the agreement between colonoscopy and pathology was almost perfect with a weighted Kappa coefficient of $0.862(95 \% \mathrm{Cl}, 0.812-0.912)$ [7].

The difference in localization between surgery and pathology was $15 \%$ (13 cases). When dividing the colon into 8 segments (cecum, ascending colon, hepatic flexure, transverse colon, splenic flexure, descending colon, sigmoid colon, and rectum), 72 cases ( $85 \%$ ) had the same location by the operative 
note and the pathology report, 11 (13\%) differed by 1 colonic segment, 1 (1\%) differed by 2 colonic segments, and 1 (1\%) differed by 3 colonic segments.

The agreement between surgery and pathology was almost perfect with a weighted Kappa coefficient of $0.938(95 \% \mathrm{Cl}$, $0.904-0.973)$. [7]

\section{Discussion}

We showed in our study that there is a $33 \%$ difference in the location of the colon mass as estimated by colonoscopy and the location seen by surgery. Fortunately, the majority of those differences ( $22 \%$ ) are within 1 colonic segment, such as colonoscopy estimated to be in the sigmoid colon and surgery estimated to be in the descending colon. However, there were $8 \%$ and $2 \%$ where the difference was of 2 and 3 colonic segments respectively, which can affect the surgical planning completely. A surgeon may be told that the patient has a descending colon mass when in fact the patient had a transverse colon lesion, which would change the surgical planning completely.

There was also a $30 \%$ difference in localization comparing colonoscopy and the surgical pathological report. The great majority (23\%) with one colonic segment difference but we observed one case (1\%) with a 5 -segment difference. It is unclear if this truly represents a difference or perhaps was a miscommunication between the endoscopist and the staff, such as someone stating "ascending colon" and the staff comprehending "descending", a common occurrence.

We were also surprised to see a $15 \%$ difference between the surgical resection specimen and the pathological localization of the lesion. There also the majority of the cases (13\%) differed by 1 colonic segment.

There have been several studies supporting the finding that colonoscopy alone is inaccurate to identify the location of the colon mass and should not be used alone for surgical planning, especially if one is planning a laparoscopic approach, where the surgeon cannot feel the colon to identify the mass [8-10]. Colonoscopy incorrectly localized the tumor in these studies between $6 \%$ and $21 \%$. Marking of the lesions during colonoscopy, i.e. endoscopic tattoing, improved the surgical localization to $98 \%$. Intraoperative colonoscopy also has a higher localization yield of $100 \%$, however, it does increase the operative time [8]. Importantly, as mentioned above, the incorrect localization can change the surgical plan completely, which can adversely affect the patients [11-14]. Yap et al reported that $4 \%$ of his patients had the surgical plans altered secondary to incorrect localization. They identified on multivariate analysis that gastroenterology training and incomplete colonoscopy were associated with an error in localization of the lesion [10]. Shah et al, reported on the use of technique called magnetic endoscope imaging (MEI), where one can obtain an image of the colonoscope inside the patient and, therefore, estimate the location of the lesion within the colon based on the location of the colonoscope, $90 \%$ accuracy of the localization using MEI using barium enema as the gold standard, which is also far from ideal [15]. Furthermore, in a recent systematic review of the literature on the topic, Acuna et al proposed the colonoscopic tat- tooing should be used for tumor localization as it has improved performance characteristics compared for magnetic endoscope imaging, radiological guidance, and pure colonoscopic guidance, improving the accuracy in approximately $5.9 \%$ with minimal side-effects [16]. Kanazawa et al, reported that tumor localization by CT colonography (CTC) seems to be superior to optical colonoscopy (OC) $90 \%$ vs. CTC at $98 \%(P<0.05)$ and invasion depth assessment (OC, $55 \%$; CTC, $73 \%$; $P<0.05)$ [17].

Our study has several limitations. It is a retrospective singlecenter study in a safety net hospital where we currently do not offer specialized colorectal surgery care. Most of the surgical resections were performed by general surgeons. The colonoscopies were performed by trainees and faculty gastroenterologists. Our trainees perform several procedures independently with faculty support but attending gastroenterologists are always available to confirm cases, especially in the case of colonic masses. We need to acknowledge this as it may be the reason why our discrepancy is higher than the published literature. Many operative reports did not specify the location of the colonic masses, which negatively impact our number of endoscopic findings to compare. Some of the pathology reports were also limited in describing specific colonic segments where lesions were found, since gross colonic samples do not always include anatomic landmarks, making it difficult to determine the exact location of the mass within the colon.

Our study also has several strengths. It is the first one to our knowledge to compare the accuracy in localization among colonoscopy, surgery, and pathology. We were able to demonstrate that even when one compare pathological and surgical localization there is a small but not negligible difference in localization of $15 \%$. We also further stratified the discrepancies and counted the number of colonic segments which can alter the surgical planning and affect patients' outcomes.

\section{Conclusion}

Our study reinforces the challenge that endoscopists face when estimating the location of colonic lesions with only a luminal view with limited anatomical landmarks. Based on the published literature, there should be routine use of methods to mark and identify anatomic locations of lesions, such as endoscopic tattooing, when one is confronted with a colonic mass or any endoscopic lesions of interest. Perhaps use of preoperative CT colonography in addition to colonoscopy with tattooing can minimize even more any localization discrepancy. Efforts should be made to improve endoscopic localization of lesions by endoscopists to accurately guide surgical planning and resection and to positively affect patient outcome.

\section{Competing interests}

None 


\section{References}

[1] Jass JR. Colorectal cancer: a multipathway disease. Crit Rev Oncol 2006; $12: 273-287$

[2] Derwinger K, Gustavsson B. Variations in demography and prognosis by colon cancer location. Anticancer Research 2011; 31: 2347-2350

[3] Jass JR. Molecular heterogeneity of colorectal cancer: Implications for cancer control. Surg Oncol 2007; 16: (Suppl. 01): S7 - S9

[4] Phipps Al, Lindor NM, Jenkins MA et al. Colon and rectal cancer survival by tumor location and microsatellite instability: the colon cancer family registry. Dis Colon Rectum 201356: 937 - 944

[5] Hemminki K, Santi I, Weires M et al. Tumor location and patient characteristics of colon and rectal adenocarcinomas in relation to survival and TNM classes. BMC Cancer 2010; 10: 688

[6] Baxter NN, Goldwasser MA, Paszat LF et al. Association of colonoscopy and death from colorectal cancer. Ann Intern Med 2009; 150: 1-8

[7] Landis JR, Koch G. The measurement of observer agreement for categorical data. Biometrics 1977; 33: 159-174

[8] Cho YB, Lee WY, Yun HR et al. Tumor localization for laparoscopic colorectal surgery. World J Surg 2007; 31: 1491-1495

[9] Saleh F, Abbasi TA, Cleghorn M et al. Preoperative endoscopy localization error rate in patients with colorectal cancer. Surg Endosc 2015; 29: $2569-2575$
[10] Yap R, lanno D, Burgess A. Colonoscopic localization accuracy for colorectal resections in the laparoscopic era. Am J Surg 2016; 212: $258-263$

[11] Benedix F, Kube R, Meyer F et al. Comparison of 17,641 patients with right- and left sided colon cancer: differences in epidemiology, perioperative course, histology, and survival. Dis Colon Rectum 2010; 53 : $57-64$

[12] Suttie SA, Shaikh I, Mullen R et al. Outcome of right- and left-sided colonic and rectal cancer following surgical resection. Colorectal Dis 2011; 13: $884-889$

[13] Meguid RA, Slidell MB, Wolfgang CL et al. Is there a difference in survival between right-versus left-sided colon cancers? Ann Surg Oncol 2008; 15: $2388-2394$

[14] Wray CM, Ziogas A, Hinojosa MW et al. Tumor subsite location within the colon is prognostic for survival after colon cancer diagnosis. Dis Colon Rectum 2009; 52: 1359-1366

[15] Shah SG, Pearson H], Moss S et al. Magnetic endoscope imaging: a new technique for localizing colonic lesions. Endoscopy 2002; 34: $11900-904$

[16] Acuna SA, Elmi M, Shah PS et al. Preoperative localization of colorectal cancer: a systematic review and meta-analysis. Surg Endosc 2016; 31: $2366-2379$

[17] Kanazawa H, Utano K, Kijima S et al. Combined assessment using optical colonoscopy and computed tomographic colonography improves the determination of tumor location and invasion depth. Asian J Endosc Surg 2016; 10: $28-34$ 